\title{
Disseminated Sporotrichosis with Osteolytic Bone Involvement
}

\author{
Paul B. Aronowitz, $\mathrm{MD}^{7}$, Meghan Gilroy, $\mathrm{MD}^{2}$, and Katerina N. Christiansen, $\mathrm{MD}^{7}$ \\ 'Department of Internal Medicine, University of California, Davis, School of Medicine, Sacramento, CA, USA; ${ }^{2}$ Division of Gastroenterology, \\ Hepatology \& Nutrition, University of Utah, Salt Lake City, Utah, USA.
}
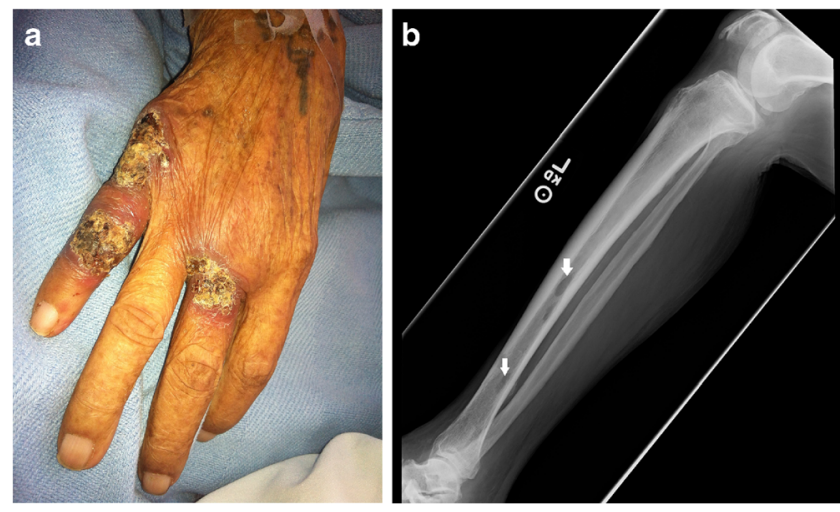

Figure 1 Right hand.

A n 89-year-old Laotian immigrant with a past medical history of diet-controlled diabetes mellitus presented with a 4month history of fevers and skin lesions that persisted despite two courses of oral antibiotics. He also complained of dysphagia and odynophagia. He denied recent travel or gardening, but reported exposure to outdoor-dwelling cats. Physical examination revealed abscesses in various stages of healing involving the chin, trunk and elbows, but were most pronounced on the fingers, hands (Fig. 1) and feet. X-rays of both lower extremities (performed to assess for osteomyelitis) revealed multiple cortical tibial lucencies consistent with disseminated mycosis (Fig. 2). Wound swab and bone cultures grew Sporothrix schenckii. Biopsies of the left false vocal cord showed granulomatous inflammation consistent with sporotrichosis. HIV testing returned negative results.

Sporotrichosis is caused by infection with the dimorphic fungus Sporothrix schenckii, and is classically transmitted via soil and plant material such as sphagnum moss; zoonotic transmission has also been reported, most commonly from cats. ${ }^{1,2}$ The most common form of sporotrichosis is cutaneous, occurring on the hand or arm inoculated by a contaminated rose thorn or cat's claw puncture. Disseminated sporotrichosis occurs most commonly in

Received December 21, 2016

Revised February 22, 2017

Accepted March 9, 2017

Published online March 27, 2017

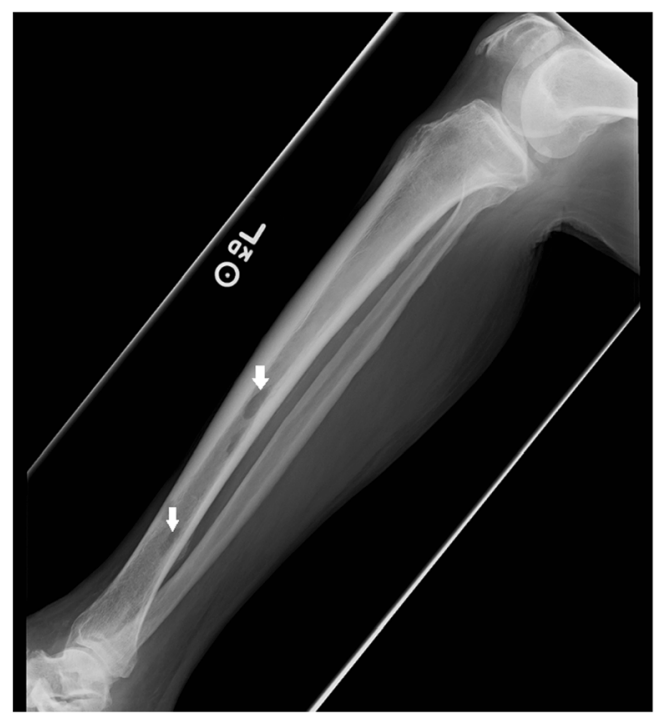

Figure 2 Radiograph of the left leg with osteolytic lesions of the tibia (arrows).

immunocompromised hosts, but this patient's likely risk factors for dissemination were his diabetes and age.

Corresponding Author: Paul B. Aronowitz, MD; Department of Internal MedicineUniversity of California, Davis, School of Medicine, 4150 V Street, Suite 3100, Sacramento, CA 95817, USA (e-mail: Paronowitz@ucdavis.edu).

\section{Compliance with Ethical Standards:}

Conflict of Interest: The authors declare that they do not have a conflict of interest.

Funding: None

\section{REFERENCES}

1. Vasquez-del-Mercado E, Arenas R, Padilla-Desgarenes C. Sporotrichosis. Clin Dermatol. 2012;30:437-43.

2. Schubach A, Schubach T, Barros M. Epidemic cat-transmitted sporotrichosis. New Engl J Med. 2005;353:1185-6. 